\title{
COEFFICIENTS OF MEROMORPHIC SCHLICHT FUNCTIONS
}

\author{
PETER L. DUREN ${ }^{1}$
}

ABstract. This paper presents an elementary proof of a known theorem on the coefficients of meromorphic schlicht functions: if $f \in \Sigma$ and $b_{k}=0$ for $1 \leqq k<n / 2$, then $\left|b_{n}\right| \leqq 2 /(n+1)$.

Let $\Sigma$ denote the class of functions

$$
f(z)=z+b_{0}+b_{1} z^{-1}+b_{2} z^{-2}+\cdots
$$

analytic and schlicht in $|z|>1$ except for a simple pole at $\infty$ with residue 1 . Let $\Sigma_{0}$ be the subclass of $\Sigma$ for which $b_{0}=0$. It follows from the area theorem that $\left|b_{1}\right| \leqq 1$, and Schiffer [7] obtained the sharp estimate $\left|b_{2}\right| \leqq 2 / 3$. The form of the extremal functions suggested that $\left|b_{n}\right| \leqq 2 /(n+1)$ for all $n$. This has proved to be quite false, but it is true for certain subclasses of $\Sigma$. For example, the following theorem goes back to Goluzin [3].

Theorem. Let $f \in \Sigma$ and suppose $b_{1}=b_{2}=\cdots=b_{m-1}=0$ for some $m \geqq 1$. Then $\left|b_{n}\right| \leqq 2 /(n+1), n=m, m+1, \cdots, 2 m$.

The inequality $\left|b_{2}\right| \leqq 2 / 3$ is a special case of this theorem. Jenkins [5] proved the theorem by the method of quadratic differentials. I claimed [1] to give an elementary proof based on the Grunsky inequalities. However, Professor Jenkins has pointed out to me that my proof contains an error, since the square-root transformation $\sqrt{ }\left(f\left(z^{2}\right)\right)$ will introduce a branch point wherever $f\left(z^{2}\right)=0$. Furthermore, Goluzin made essentially the same mistake (see the footnote in $[4$, p. 279]).

The purpose of this paper is to correct the error in [1] and thus to deduce the theorem in an elementary way from the Grunsky inequalities. The main idea was suggested by Pommerenke's recent proof [6] that $\left|b_{2}\right| \leqq 2 / 3$.

The $n$th Faber polynomial $F_{n}(w)$ of a function $f \in \Sigma$ is defined by

$$
F_{n}[f(z)]=z^{n}+\sum_{k=1}^{\infty} \beta_{n k} z^{-k}
$$

Received by the editors March 16, 1970.

AMS 1970 subject classifications. Primary 30A34.

Key words and phrases. Coefficient estimates, schlicht functions, Faber polynomials, Grunsky inequalities.

${ }^{1}$ Research supported in part by National Science Foundation Grant GP-19148. 
The inequality

$$
\left|\beta_{n n}\right| \leqq 1, \quad n=1,2, \cdots,
$$

is a very special case of the Grunsky inequalities.

To prove the theorem, it is enough to show $\left|b_{2 m-1}\right| \leqq 1 / m$ and $\left|b_{2 m}\right| \leqq 2 /(2 m+1)$. For the first of these inequalities, assume without loss of generality that $f \in \Sigma_{0}$ and observe (as in [1]) that

$$
[f(z)]^{m}=F_{m}[f(z)]=z^{m}+\sum_{j=1}^{\infty} \beta_{m j} z^{-j},
$$

with $\beta_{m m}=m b_{2 m-1}$. Hence $\left|b_{2 m-1}\right| \leqq 1 / m$, by (1).

To obtain the estimate for $\left|b_{2 m}\right|$, assume $b_{0}$ is chosen so that $f(z) \neq 0$ in $|z|>1$, and let

$$
g(z)=\sqrt{ }\left(f\left(z^{2}\right)\right)=z\left\{1+b_{0} z^{-2}+b_{m} z^{-2 m-2}+b_{m+1} z^{-2 m-4}+\cdots\right\}^{1 / 2} .
$$

Let $F_{n}^{*}(w)$ be the $n$th Faber polynomial of $g$, so that

$$
F_{n}^{*}[g(z)]=z^{n}+\sum_{j=1}^{\infty} \beta_{n j}^{*} z^{-j}
$$

The proof depends on the following lemma.

LEMma. For each fixed $m$, there exist real numbers $r_{k}$ such that

$$
\beta_{2 k+1,2 m+1}^{*}=((2 k+1) / 2) b_{m+k}+r_{k} b_{0}^{m+k+1}, \quad k=0,1, \cdots, m .
$$

Proof. The lemma will be proved by induction on $k$. For $k=0$, it is clear that $F_{1}[g(z)]=g(z)$, and one computes

$$
\beta_{1,2 m+1}^{*}=\frac{1}{2} b_{m}+\left(\begin{array}{c}
\frac{1}{2} \\
m+1
\end{array}\right) b_{0}^{m+1},
$$

where $\left(\begin{array}{l}x \\ n\end{array}\right)=x(x-1) \cdots(x-n+1) / n$ ! is a binomial coefficient. Assuming now that the lemma has been proved for $0,1, \cdots, k-1$, consider

$$
\begin{aligned}
{[g(z)]^{2 k+1}=} & z^{2 k+1}\left\{1+b_{0} z^{-2}+b_{m} z^{-2 m-2}+\cdots\right\}^{k+1 / 2} \\
= & z^{2 k+1} \sum_{j=0}^{\infty}\left(\begin{array}{c}
k+\frac{1}{2} \\
j
\end{array}\right)\left(1+b_{0} z^{-2}\right)^{k-j+1 / 2}\left(b_{m} z^{-2 m-2}+\cdots\right)^{j} \\
= & \left(1+b_{0} z^{-2}\right)^{k+1 / 2} z^{2 k+1}+\left(k+\frac{1}{2}\right)\left(1+b_{0} z^{-2}\right)^{k-1 / 2} \\
& \cdot\left(b_{m}+b_{m+1} z^{-2}+\cdots\right) z^{2(k-m)-1}+O\left(z^{-2 m-2}\right) \\
= & z^{2 k+1}+\sum_{j=1}^{k}\left(\begin{array}{c}
k+\frac{1}{2} \\
j
\end{array}\right) b_{0}^{j} z^{2(k-j)+1}+\sum_{j=1}^{\infty} \gamma_{k j} z^{-j},
\end{aligned}
$$


say. A calculation gives

(2) $\gamma_{k, 2 m+1}=\left(\begin{array}{c}k+\frac{1}{2} \\ k+m+1\end{array}\right) b_{0}^{k+m+1}+\left(k+\frac{1}{2}\right) \sum_{n=0}^{k}\left(\begin{array}{c}k-\frac{1}{2} \\ n\end{array}\right) b_{0}^{n} b_{m+k-n}$.

From the form of $[g(z)]^{2 k+1}$, it is clear that

$$
F_{2 k+1}^{*}[g(z)]=[g(z)]^{2 k+1}-\sum_{j=0}^{k-1}\left(\begin{array}{l}
k+\frac{1}{2} \\
k-j
\end{array}\right) b_{0}^{k-j} F_{2 j+1}^{*}[g(z)] .
$$

Thus by (2) and our inductive hypothesis, we have

$$
\begin{aligned}
\beta_{2 k+1,2 m+1}^{*} & =\gamma_{k, 2 m+1}-\sum_{j=0}^{k-1}\left(\begin{array}{c}
k+\frac{1}{2} \\
k-j
\end{array}\right) b_{0}^{k-j}\left[\left(j+\frac{1}{2}\right) b_{m+j}+r_{j} b_{0}^{m+j+1}\right] \\
& =\left(k+\frac{1}{2}\right) b_{m+k}+r_{k} b_{0}^{m+k+1},
\end{aligned}
$$

since

$$
\left(k+\frac{1}{2}\right)\left(\begin{array}{l}
k-\frac{1}{2} \\
k-j
\end{array}\right)=\left(j+\frac{1}{2}\right)\left(\begin{array}{l}
k+\frac{1}{2} \\
k-j
\end{array}\right), \quad j=0,1, \cdots, k-1 .
$$

This proves the lemma.

In view of (1), the lemma gives the inequality

$$
\left|((2 m+1) / 2) b_{2 m}+r_{m} b_{0}^{2 m+1}\right| \leqq 1
$$

for the case in which $f(z) \neq 0$ in $|z|>1$. Now let

$$
f(z)=z+b_{m} z^{-m}+b_{m+1} z^{-m-1}+\cdots \in \Sigma_{0},
$$

and let $E$ be the complement of the range of $f$. If $0 \in E$, then (3) gives at once the inequality $\left|b_{2 m}\right| \leqq 2 /(2 m+1)$. The proof is complete also if $r_{m}=0$.

Suppose next that $0 \notin E$ and $r_{m} \neq 0$. Assume without loss of generality that $b_{2 m}>0$. If $\alpha \in E$, then $[f(z)-\alpha]$ has no zeros in $|z|>1$, so by (3),

$$
b_{2 m} \leqq(2 /(2 m+1))\left[1+r_{m} \operatorname{Re}\left\{\alpha^{2 m+1}\right\}\right], \quad \alpha \in E .
$$

The proof will be complete, then, if we show that $\alpha \in E$ can be chosen so that

$$
r_{m} \operatorname{Re}\left\{\alpha^{2 m+1}\right\} \leqq 0 .
$$

But if this expression is positive for all $\alpha \in E$, then since $E$ is connected and $0 \notin E$, it follows that $E$ lies entirely in an open sector with vertex 0 and angle $\pi /(2 m+1)$. On the other hand, 0 is in the convex hull of $E$, 
since $\int_{0}^{2 \pi} f\left(r e^{i \theta}\right) d \theta=0, r>1$. This contradiction shows that $\alpha \in E$ can be chosen to satisfy (4), proving that $b_{2 m} \leqq 2 /(2 m+1)$.

Garabedian and Schiffer $[2]$ found that $\operatorname{Re}\left\{b_{3}\right\}$ is maximized by a function $f \in \Sigma$ with

$$
f(z)=z+4 i e^{-8} z^{-1}+\left(\frac{1}{2}+e^{-6}\right) z^{-3}+\cdots .
$$

However, the above theorem shows that $\left|b_{3}\right| \leqq \frac{1}{2}$ if $b_{1}=0$. This can be generalized as follows.

TheOREM. If $f \in \Sigma$ and $\left|\arg \left\{b_{3}\right\}-\arg \left\{b_{1}^{2}\right\}\right| \leqq \pi / 2$, then $\left|b_{3}\right| \leqq \frac{1}{2}$.

PRoof. Assume that $b_{0}=0$ and, after a suitable rotation, that $b_{3}>0$ and $\operatorname{Re}\left\{b_{1}^{2}\right\} \geqq 0$. Observe that

$$
[f(z)]^{2}=z^{2}+2 b_{1}+2 b_{2} z^{-1}+\left(2 b_{3}+b_{1}^{2}\right) z^{-2}+\cdots .
$$

Hence by (1), $\operatorname{Re}\left\{2 b_{3}+b_{1}^{2}\right\} \leqq 1$, which shows $b_{3} \leqq \frac{1}{2}$.

Similar generalizations can be made to higher coefficients.

\section{REFERENCES}

1. P. L. Duren, Coefficient estimates for univalent functions, Proc. Amer. Math. Soc. 13 (1962), 168-169. MR 28 \#1286.

2. P. R. Garabedian and M. Schiffer, $A$ coefficient ineguality for schlicht functions, Ann. of Math. (2) 61 (1955), 116-136. MR 16, 579.

3. G. M. Goluzin, Some estimates of the coefficients of schlicht functions, Mat. Sb. 3 (1938), 321-330. (Russian)

4. - On p-valent functions, Mat. Sb. 8(50) (1940), 277-284. (Russian) MR 2, 185.

5. J. A. Jenkins, On certain coefficients of univalent functions. II, Trans. Amer. Math. Soc. 96 (1960), 534-545. MR 23 \#A309.

6. Ch. Pommerenke, Unpublished Lecture Notes, March 1969.

7. M. Schiffer, Sur un probième d'extrémum de la représentation conforme, Bull. Soc. Math. France 66 (1938), 48-55.

University of Michigan, ANn Arbor, Michigan 48104 